\title{
Towards a Generic Model for Situational Method Engineering
}

\author{
Jolita Ralyté ${ }^{1}$, Rébecca Deneckère ${ }^{2}$, and Colette Rolland ${ }^{2}$ \\ ${ }^{1}$ CUI, University of Geneva, 24, bd. du Général Dufour, CH-1211 Geneva, Switzerland \\ ralyteacui.unige.ch \\ ${ }^{2}$ CRI, University of Paris Sorbonne, 90 rue de Tolbiac, 75013 Paris, France \\ \{denecker, rolland\}@univ-paris1.fr
}

\begin{abstract}
The work presented in this paper is related to the area of Situational Method Engineering (SME) which focuses on project-specific method construction. We propose a generic process model supporting the integration of different existing SME approaches. This model shall help the method engineer either selecting one SME approach or combining several approaches that best fit the situation of the method engineering project at hand. The generic model presented in this paper already contains three SME techniques: (1) to assemble method chunks (2) to extend an existing method and (3) to generate a method by abstraction/instantiation of a model/meta-model. The paper presents and illustrates these three techniques and show how other SME techniques could be integrated in the model.
\end{abstract}

\section{Introduction}

The need for a better productivity of system engineering teams, as well as a better quality of products motivates the development of solutions to adapt methods to the project situation at hand. This is known as Situational Method Engineering (SME). Whereas SME promotes the construction of a method by assembling reusable method fragments stored in some method base [9, 3, 18, 19, 23, 29, 26], in this paper we propose a generic process model to capture a large variety of approaches supporting "on the fly" method construction.

As the selection of the suitable method engineering approach for the project at hand is not easy, our generic process model guides the method engineer in the definition of his/her project method engineering goal and in the selection of the approach which best allow him/her to achieve it. Besides, as in some cases a combination of several approaches could be the most suitable engineering solution in order to construct or adapt a method, the generic process model guides the method engineer in selecting the appropriated set of approaches.

We use a strategic process meta-model called Map [1,27] to represent our generic SME process model. Map provides a representation system based on a nondeterministic ordering of intentions and strategies. A map is a labeled directed graph with intentions as nodes and strategies as edges between intentions. A triplet $<$ source intention, target intention, strategy $>$ in the map is called a section. Each section is defined by an intention achievement guideline, which provides advice to fulfil the target intention following the strategy given the source intention has been achieved. 
The directed nature of the graph shows which intentions can follow which one. Since, many strategies can be used for achieving an intention, Map allows us to integrate different approaches as different method engineering strategies in the same SME process model and to combine the application of these approaches in the construction of a new method or the adaptation of a given one.

In previous papers we presented a process model for assembly based situational method engineering [19], an approach for method extension based on the use of patterns [5,6] and more recently, an abstraction based approach [24] responding to the needs of a large method engineering project in industry. All these approaches use different techniques for method construction but their objective is the same - to support the construction of a method matching the requirements of a given situation.

Our belief is that it will be useful to investigate the problem of integrating different approaches in a single SME process model. This is the objective we aim at in this paper. We propose a generic SME process model which integrates the three abovementioned approaches. We also show that this model is flexible enough to integrate other approaches than the three that are integrated and illustrated in this paper.

The paper is organised as follows: section 2 describes our generic process model for SME. Sections 3, 4 and 5 present and illustrate respectively three method engineering approaches, namely Assembly-based, Extension-based and Paradigmbased, all being integrated in our generic model. Section 6 considers the similarities and the differences of these approaches and the possibility of their parallel use in the construction of a single method whereas section 7 draws some conclusions and discuss about our future work.

\section{Generic Process Model for Situational Method Engineering}

We consider that any SME process is made of two main tasks: setting the method engineering goal and then, constructing a method that matches this goal. In other words, there are two core intentions that the method engineer has in mind:

1. Set Method Engineering Goal that is to identify the kind of method he/she needs,

2. Construct a Method allowing him/her to satisfy this goal.

These intentions are the nodes of the map presented in Fig.1. As shown in this figure, the Map representation formalism allows us to propose several different strategies to support the realisation of these intentions.

The achievement of the first intention, namely Set Method Engineering Goal, depends on the method situation of the project at hand. In one project situation the method engineer may perhaps consider that a specific method could be applicable but requires some adaptations whereas in other situations, he or she may be convinced that any of the available methods is suitable for the project. In the first case, the method engineering (ME) goal refers to the adjustments of the selected method: enhancement, extension or restriction. We call the corresponding strategy the Methodbased strategy. In the second case, it is necessary to construct a completely new method and the corresponding strategy is called the From scratch strategy.

The achievement of the intention Construct a Method depends on the applied method construction technique. Thanks to the map structure it is easy to integrate in the same model different method construction techniques as different strategies to reach the intention Construct a Method. The map of Fig. 1 proposes three SME 
techniques. The first one is based on the reuse of method components extracted from existing methods and stored in some method base. This technique helps to select and assemble different method components in order to construct a new method or to enrich an existing one. As a consequence, the corresponding strategy is called Assembly-based strategy. The second technique is used for extending a method by applying extension patterns and therefore it is referred in the map by the Extensionbased strategy. Finally, the third technique is relevant when a new fresh method must be constructed either by abstracting from a given model or by instantiating a metamodel. This new method is based on some paradigm model or meta-model and this is why the corresponding strategy is called the Paradigm-based strategy. It is obvious that these three strategies can be combined in order to construct the method best fitting the situation of the project at hand.

Once the required method has been constructed it is necessary to validate it. For this, we propose the Evaluation strategy which implements different method evaluation techniques. Our Generic Process Model for SME is shown in Fig. 1.

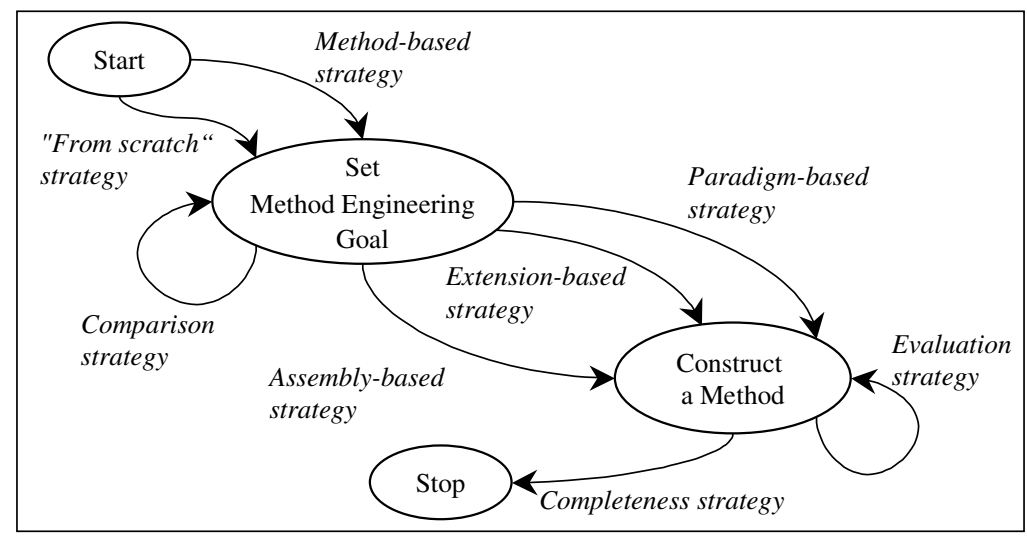

Fig. 1. Generic Process Model for Situational Method Engineering

It is obvious, that other SME techniques could be integrated in our generic SME process model as other strategies to achieve the intention Construct a Method. In this paper we concentrate on the three method construction techniques introduced in the map above and we present and illustrate them in the following sections.

\section{Assembly-Based Method Engineering}

Our approach for assembly-based SME aims at constructing a method 'on the fly' in order to match as well as possible the situation of the project at hand. It consists in the selection of method components (that we call method chunks) from existing methods that satisfy some situational requirements and their assembly.

Our approach is requirements-driven, meaning that the method engineer must start by eliciting requirements for the method. Next, the method chunks matching these 
requirements can be retrieved from the method base. And finally, the selected chunks are assembled in order to compose a new method or to enhance an existing one.

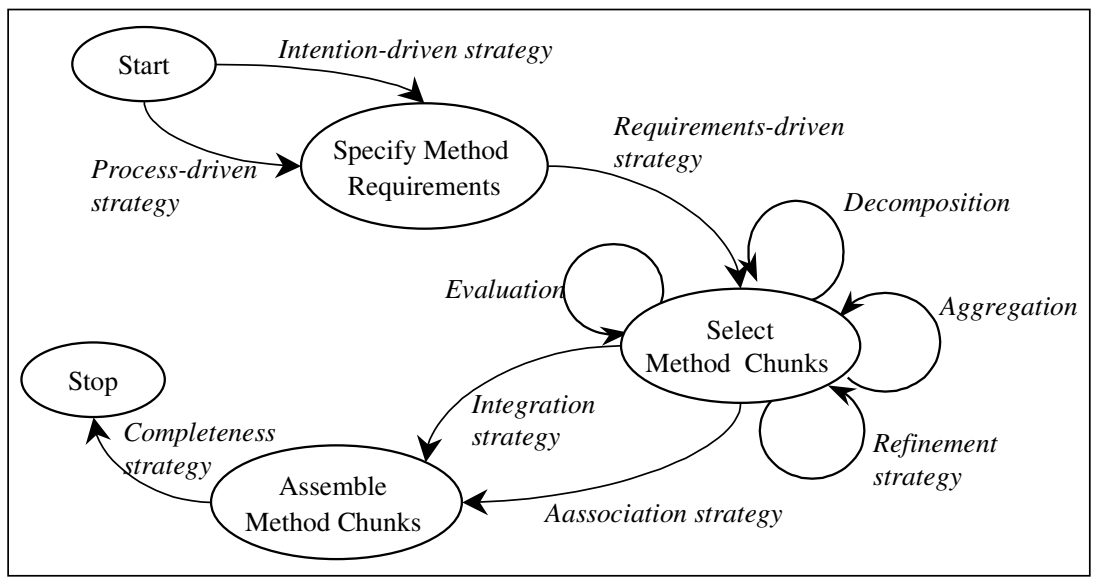

Fig. 2. Assembly-Based Process Model for Situational Method Engineering

As a consequence, the three key intentions in the assembly-based method engineering process are: Specify Method Requirements, Select Method Chunks and Assemble Method Chunks. Fig. 2 depicts our assembly-based process model for SME.

\subsection{Method Requirements Specification}

The elicitation of method requirements must be done in the light of the method engineering goal set previously (see Fig.1) that might be to adapt an existing method or to construct a new one. We identified two different strategies for requirements elicitation, namely Intention-driven strategy and Process-driven strategy (Fig. 2).

The first strategy is suitable for method adaptation. There are different types of method adaptation. The method in use can be strong in terms of its product model but weak with respect to its process model, which will be the subject of adaptation and enhancement. The adaptation can be to simply add a new functionality to the existing method, which is relevant in its other aspects. Vice versa, the project at hand could not need some functionality offered by the method. In all these cases, the requirements elicitation process is driven by the identification of the ME intentions such as 'add event concept', 'complete completeness checking step' etc., which will allow to complete, enhance or limit the method initially selected. For this reason, wee call this strategy Intention-driven strategy (Fig. 2).

The second strategy is relevant in the case of a new method construction. In such $\mathrm{ME}$ situation the requirements is not only to produce the list of $\mathrm{ME}$ intentions that will permit to adapt the selected method but to identify the full set of engineering intentions that shall be fulfilled by the new method. For this reason we call the corresponding strategy Process-driven strategy. 
Both of these strategies lead to a set of requirements expressed as a map that we call the requirements map. More information about the guidelines supporting these two strategies to Specify Method Requirements can be found in [21].

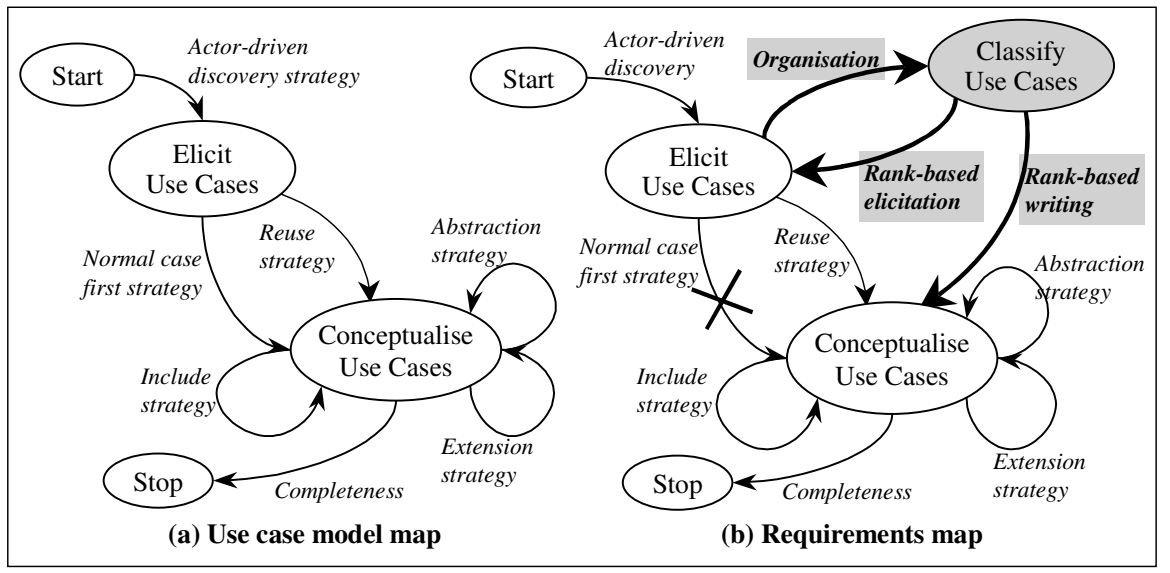

Fig. 3. Requirements Map for the Use Case Model Enhancement

As an example, let's assume that the goal of the method engineer is to enhance the use case approach proposed by Jacobson in [11] by some scenario classification \& authoring guidelines in order to improve the quality of use case conceptualisation. This is a case of method adaptation and therefore, the Intention-driven strategy is the most suitable one. This strategy supports the identification of adaptation requirements expressed as intentions and strategies as shown in Fig. 3. The initial map for use case construction is shown in Fig. 3 (a) whereas Fig. 3 (b) highlights the requirements for enhancement expressed through three new sections (in colour) and the deletion of the Normal case first strategy which is replaced by the added features.

\subsection{Method Chunks Selection}

Once the method requirements have been specified, the selection of the method chunks matching these requirements can start. The Requirements-driven strategy helps the method engineer to select the best fitting chunks. The chunk selection queries must be formulated by giving values to the attributes of the descriptors and interfaces of method chunks (see $[15,23]$ ). The validation of the retrieved chunks is supported by the Evaluation strategy which helps in evaluating the degree of matching of the candidate chunk to the requirements. This is done by applying similarity measures between the requirements map and the process model of the selected chunk. More details about these similarity measures could be found in [19].

The Decomposition, Aggregation and Refinement strategies help to refine the candidate chunk selection by analysing more in depth if the chunk matches the requirements. If the selected method chunk is an aggregate one, i.e. it is composed of several chunks, the Decomposition strategy drives the selection of the relevant sub- 
chunks and the elimination of the inadequate ones. Vice-versa, the Aggregation strategy is applicable when the retrieved chink matches partly the requirements. This strategy proposes to look for an aggregate chunk containing the candidate one based on the assumption that the aggregate chunk might provide a solution for the missing requirements. The Refinement strategy proposes to search for another chunk satisfying the same intention but providing different guidelines to achieve it. In our example, the query for the method chunk selection will include parameters as follows:

Application domain = 'Information System' AND Design activity

= 'Requirements specification' AND Situation = 'Use cases'

AND Intention = 'Classify use cases'

\subsection{Method Chunks Assembly}

When at least two chunks have been selected, the method engineer can progress in the assembly of these chunks following one of the two proposed strategies, namely the Association strategy and the Integration strategy (Fig. 2).

The Association strategy is relevant when the method chunks to assemble correspond to two different system engineering functionalities, i.e. they allow to achieve different engineering intentions and the result of one chunk is used as a source product by the second one. Such method chunks generally do not have common elements in their product and process models and the assembly process is therefore mainly dealing with making the bridge between the two chunks. More precisely, the product models of the chunks must be connected by defining links between their different concepts whereas the connection of their process models consists in defining their execution order. The connection of the product model is possible thanks to the ADD_LINK and ADD_CONCEPT operators [22]. The MERGE_INTENTION operator is applied to connect the process models and consists in identifying in the first chunk the intention producing the source product for the second chunk. Some method chunk adaptation could be required before their assembly in order to avoid concepts name ambiguity. This may be done by applying different RENAME operators. For example, the chunk producing use cases and the chunk constructing the system object structure can be assembled to get a method with a larger coverage than any of the two initial ones.

The Integration strategy is relevant to assemble chunks that have similar engineering goals but provide different ways to satisfy it. In such a case, the process and product models are overlapping, that is containing the same or similar elements. The assembly process consists in identifying the common elements in the chunks product and process models and merging them. Therefore, the integration process is mainly based on the application of different MERGE operators as MERGE_ INTENTION, MERGE-SECTION for the integration of process models and MERGE_CONCEPT, MERGE_LINK for the integration of product models. Each of these operators deals with the integration of similar elements belonging to the initial chunks into a new one in the integrated chunk. The SPECIALISE and GENERALISE operators define respectively the specialisation and generalisation links between the concepts of the chunks product models. Their application is useful to build a model of the integrated method chunk that is richer than those of the initial chunks. 
Like in the previous case, the adaptation of the method chunks must precede their integration. Besides the RENAME operators, OBJECTIFY_LINK and OBJECTIFY_ PROPERTY may be required for performing more complex transformation tasks.

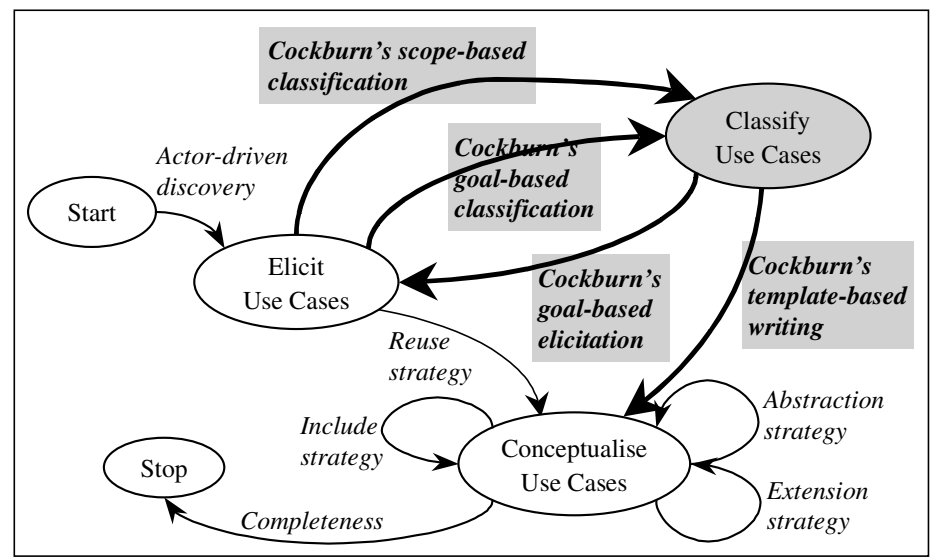

Fig. 4. The Enhanced Use Case Model Map.

For instance, the Integration strategy will be necessary to satisfy the method requirements defined in Fig. 3 as the method engineering objective is to enhance the use case conceptualisation process by new way of working. Let's suppose that the method engineer selects the use case conceptualisation guidelines proposed by Cockburn [4]. This approach proposes two complementary use case classification techniques: one is based on a three level goal hierarchy; other defines a design scope to capture in a use case typology. These two techniques cover the section $<$ Elicit Use Cases, Classify Use Cases, Organisation strategy $>$ in the requirements map. The guidelines supporting elicitation of other use cases of the lower or higher abstraction level are also provided by this approach and cover the section <Classify Use Cases, Elicit Use Cases, Rank-based elicitation strategy. Moreover, this approach proposes different templates for use case writing as well as the content guidelines depending on the use case goal level and design scope. It covers the section <Classify Use Cases, Conceptualise Use Cases, Rank-based writing strategy>. The obtained method (Fig. 7) will provide guidelines richer than those of each chunk used separately.

\section{Extension-Based Method Engineering}

Our approach for extension-based SME [5, 6] corresponds to a ME goal to adapt locally a method to the contingency of the project at hand. The approach guides method engineer by providing extension patterns that help identifying typical extension situations and provide advises to perform the required extension.

Fig. 5. shows the map representing the process underlying this approach. It can be seen that we advocate two different ways to extend a method: (a) directly through the 


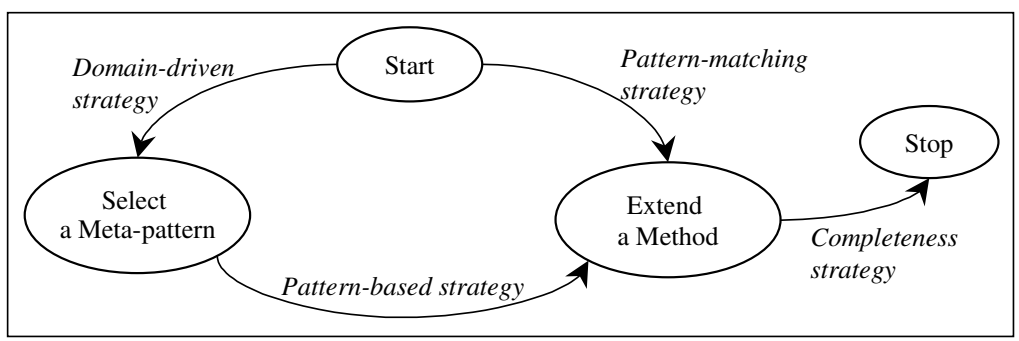

Fig. 5. Extension-Based Process Model for Situational Method Engineering

Pattern-matching strategy or (b) by using some generic knowledge related to the domain for which the extension is to be done through the path Select a Meta-pattern, Extend a Method with the Pattern-based strategy. The former helps to match extension patterns stored in a library to the extension requirements whereas the latter selects first, a meta-pattern corresponding to the extension domain and then, guides the method extension by applying the patterns suggested by the meta-pattern.

Both ways-of-working use a library of extension patterns but do it in different ways. The domain centric way exploits the fact that a set of patterns and their use can be embodied in a meta-pattern that is suitable for method extension in this domain (e.g. temporal data structures). If the required extension does not clearly correspond to a certain type of extension, a well-identified extension domain, then the pattern matching approach shall be selected by the method engineer.

\subsection{Domain-Driven Adaptation}

This path in the process of Fig. 5 comprises to Select a Meta-pattern with the Domain-driven strategy and then, to Extend a Method with the Pattern-based strategy. The guideline to Select a Meta-pattern helps the method engineer in recognising if the extension he/she has in mind corresponds to one of the so-called extension domains. A representative example of such domain is temporal data structures. The adaptation of a method to this domain includes different extensions such as the integration into the method product model the selected time model defining the appropriated calendar(s) (Gregorian, week-based, working-day-based etc.), the integration of temporal events and temporal expressions for defining event occurrence conditions etc. These extensions are captured in different patterns and organised in a meta-pattern. Meta-patterns for the different domains, for which knowledge about the extension and how to perform it has been defined, are stored in a repository together with the corresponding patterns.

Once the method engineer has selected the meta-pattern relevant for the extension domain at hand, the pattern-based strategy guides him/her in the systematic application of the patterns associated to the meta-pattern. Table 1 shows the metapattern guiding method extension with temporal data structures. 
Table 1. Meta-pattern "Extend a method with temporal concepts"

\begin{tabular}{|c|c|c|}
\hline Concept & Pattern & Arguments \\
\hline $\begin{array}{l}\text { Temporal } \\
\text { Event }\end{array}$ & $\begin{array}{l}\text { Extend a } \\
\text { method with } \\
\text { temporal events }\end{array}$ & $\begin{array}{l}\text { Applications often need to trigger operations following a specific } \\
\text { time data. This pattern allows the insertion of temporal events in } \\
\text { the method. They use a time model and the temporal domains in } \\
\text { order to define the specific moment to trigger the operations. }\end{array}$ \\
\hline $\begin{array}{l}\text { Time } \\
\text { model } \\
\text { with } \\
\text { discrete } \\
\text { time point }\end{array}$ & $\begin{array}{l}\text { Extend a } \\
\text { method with a } \\
\text { discrete time } \\
\text { point data } \\
\text { structure }\end{array}$ & $\begin{array}{l}\text { Temporal databases need to use precise knowledge on events. } \\
\text { This pattern allows defining a time model (linear or having a tree } \\
\text { structure) defined as a set of time points (or instants). These } \\
\text { points have an asymmetric and transitive time precedence } \\
\text { relationship. }\end{array}$ \\
\hline $\begin{array}{l}\text { Time } \\
\text { model } \\
\text { with } \\
\text { interval }\end{array}$ & $\begin{array}{l}\text { Extend a } \\
\text { method with } \\
\text { temporal types }\end{array}$ & $\begin{array}{l}\text { Some applications need to manage fuzzy or imprecise temporal } \\
\text { data. This pattern allows defining a time model seen as a set of } \\
\text { intervals. It helps to describe three temporal types: instants, } \\
\text { intervals, and periods, defined to manipulate the time. }\end{array}$ \\
\hline $\begin{array}{l}\text { Temporal } \\
\text { domain }\end{array}$ & $\begin{array}{l}\text { Extend a } \\
\text { method with } \\
\text { temporal } \\
\text { domains }\end{array}$ & $\begin{array}{l}\text { Classic methods mostly use the Date, Time and Time zone } \\
\text { domains linked to an attribute. However, these domains are very } \\
\text { poor and do not allow the representation of relative or periodic } \\
\text { attribute. This pattern aims at supporting the generation of these } \\
\text { temporal domains. The time used may be valid, transaction or } \\
\text { user-defined [30]. }\end{array}$ \\
\hline $\begin{array}{l}\text { Object } \\
\text { history }\end{array}$ & $\begin{array}{l}\text { Extend a } \\
\text { method with } \\
\text { temporal classes } \\
\text { for object } \\
\text { histories }\end{array}$ & $\begin{array}{l}\text { Histories are sometimes required by applications in order to look } \\
\text { at the data evolution and to execute the replay functions required } \\
\text { for tracking decisional process of an organization. Therefore, the } \\
\text { application should provide information for each object state when } \\
\text { it is/was true as well as when it is/was exploitable. This pattern } \\
\text { permits to integrate time management into the class definition and } \\
\text { to group properties that evolve at the same time and that are } \\
\text { linked to the same temporal dimension. }\end{array}$ \\
\hline $\begin{array}{l}\text { Object } \\
\text { versioning }\end{array}$ & $\begin{array}{l}\text { Extend a } \\
\text { method with } \\
\text { temporal classes } \\
\text { for object } \\
\text { versioning }\end{array}$ & $\begin{array}{l}\text { Rollback operations are required in order to come back to } \\
\text { previous states of the database. This pattern permits the creation } \\
\text { of object histories supporting the application of rollback } \\
\text { operations without endangering the database coherency. } \\
\text { Documentation operations help the engineer to keep track of the } \\
\text { different versions. }\end{array}$ \\
\hline $\begin{array}{l}\text { Time } \\
\text { constraint }\end{array}$ & $\begin{array}{l}\text { Extend a } \\
\text { method with } \\
\text { time constraint }\end{array}$ & $\begin{array}{l}\text { The necessity to handle time introduces another problem that is to } \\
\text { constrain data evolution. Models must include concepts helping to } \\
\text { define which constraints are related to the time in order to keep } \\
\text { the data coherency. This pattern proposes two constraints } \\
\text { classification: (1) intra-object and inter-object constraints [7] and } \\
\text { (2) intra-time and inter-time constraints [2]. }\end{array}$ \\
\hline
\end{tabular}

\subsection{Pattern-Matching Based Extension}

The Pattern-matching strategy to Extend a Method (Fig. 5) helps in the selection of the extension patterns which better match requirements. Therefore, the process map representing this approach (Fig. 6) is centred around two core intentions: Specify Extension Requirements and Select \& Apply a Pattern.

The Requirement elicitation strategy helps the method engineer to construct a map representing the method extension requirements. This map is called requirements map. Introduce time model could be an intention (a requirement) in the requirements map; Interval based could be a strategy to achieve this intention. Discrete time point could be another strategy to reach the same intention. These two manners to Introduce 


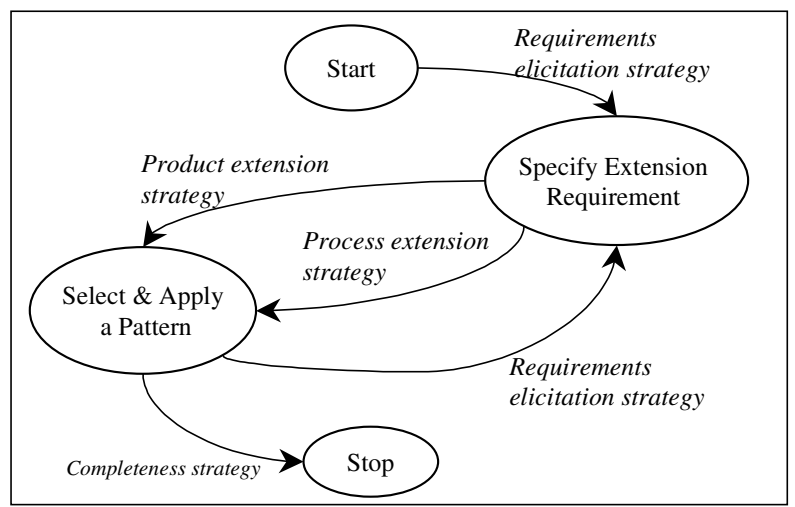

Fig. 6. Process Model for Pattern-Matching Based Method Extension

time model can be defined in two different requirements maps or can be captured in the same map meaning that the method extension will handle both of them.

Our extension patterns are divided into product extension patterns and process extension patterns. The former indicate how to extend a product model whereas the latter deal this the process model extension. This is reflected in the map of Fig. 6 by two strategies to Select \& Apply a Pattern namely the Product extension strategy and the Process extension strategy. Both of them are supported by the guidelines which help to match the requirements map intentions and strategies with the pattern situation. Some of the similarity measures introduced in section 3 are applicable here to help in finding the 'best' fit between the requirements and the pattern situation.

\section{$5 \quad$ Paradigm-Based Method Engineering}

This approach uses meta-modelling as its underlying method engineering technique. It is the most generic of the three approaches to Construct a Method that we propose in our generic model shown in Fig. 1. The hypothesis of this approach is that the new method is obtained either by abstracting from an existing model or by instantiating a meta-model. We call this starting model the paradigm model.

Meta-modelling is known as a technique to capture knowledge about methods. It is a basis for understanding, comparing, evaluating and engineering methods. One of the results obtained by the meta-modelling community is the definition of any method as composed of a product model and a process model [16]. The product model defines the set of concepts, their properties and relationships that are needed to express the outcome of the process. The process model comprises the set of goals, activities and guidelines to support process goal achievement and action execution. Therefore, method construction following the meta-modelling technique is centred on the definition of these two models. This is reflected in the paradigm-based process map by the two core intentions Construct a Product model and Construct a Process model (Fig. 7). A number of product meta-models [8, 10, 17, 28] as well as process metamodels $[12,25,27]$ are available and our approach is based on some of them. 


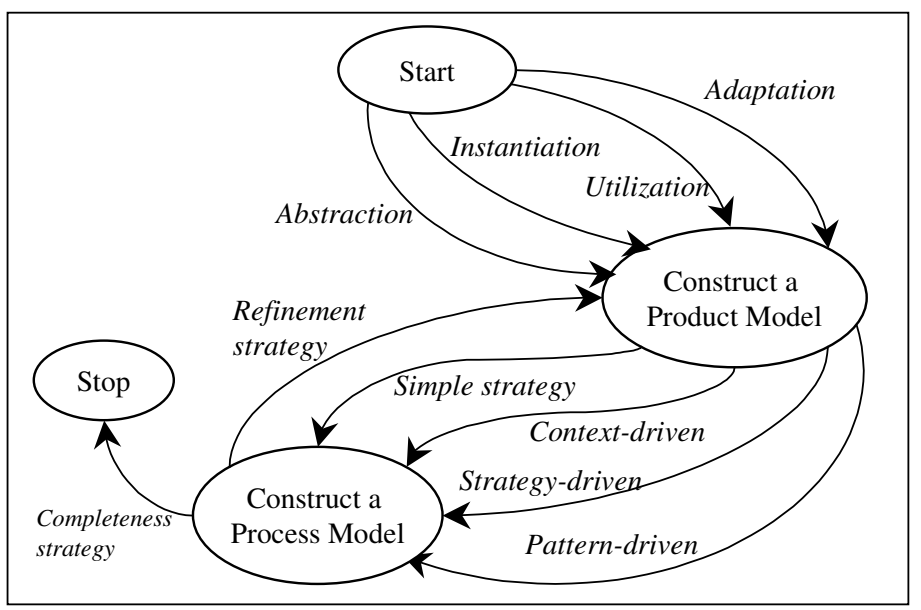

Fig. 7. Paradigm-Based Process Model for Situational Method Engineering

The construction of the product model depends of the method engineering (ME) goal. For example, the ME goal could be to construct a method:

- by raising (or lowering) the level of abstraction of a given model,

- by instantiating a selected meta-model,

- by adapting a meta-model to some specific circumstances,

- by adapting a model.

Each of these cases defines a strategy to Construct a Product model, namely the Abstraction, Instantiation, Adaptation and Utilisation strategies. Each of them is supported by a guideline. For example, the map of Fig. 8 expresses the guideline supporting the Abstraction strategy. According to this guideline, the product model construction consists in defining different product model elements such as objects, links and properties. It starts by the abstraction of some elements from the paradigm model. After that, the model under construction is refined thanks to the Generalisation, Specialisation, Aggregation, Decomposition and Transformation strategies.

The process model definition must conform to the product model. This is the reason why in the map of Fig. 7 the intention Construct a Process model follows the one to Construct a Product model. We know that a process model can take multiple different forms. It could be a simple informal guideline, a set of ordered actions or activities to carry out, a set of process patterns to be followed or a multi-process guideline combining several different alternative ways of working. These four cases are represented in our paradigm-based process model by four strategies: Simple, Context-driven, Pattern-driven and Strategy-driven. Simple and Context-driven process models are based on the NATURE process modelling formalism [12, 25] whereas the Strategy-driven process model, also called Map formalism, was proposed by [27] (see the introduction of this paper). The guidelines supporting the application of these strategies can be found in $[1,20]$. 


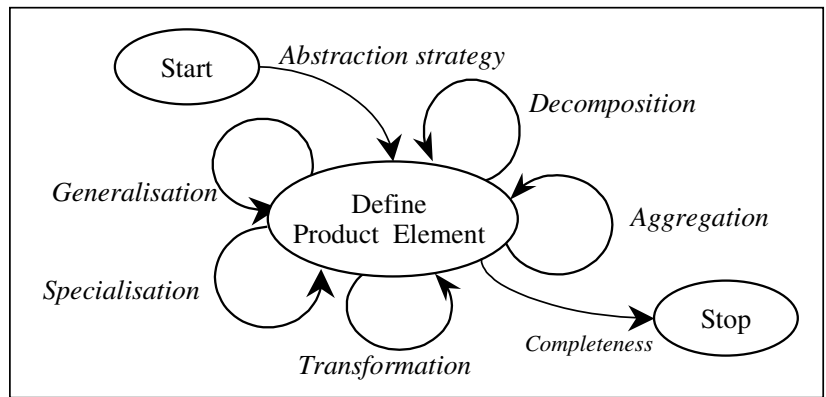

Fig. 8. Abstraction Strategy for Product Model Construction

Fig. 9 is an illustration of the use of the Abstraction strategy to Construct a Product model in the Lyee ${ }^{1}$ industrial project. The guideline of Fig. 8 was followed for the definition of the Lyee User Requirements Model (LURM) by abstracting from the Lyee Software Requirements Model (LSRM) [24]. The latter is used by the LyeeAll CASE tool $[13,14]$ in order to generate programs, provided a set of wellformatted software requirements are given. These requirements are expressed in rather low-level terms such as screen layouts and database accesses. Moreover they are influenced by the LyeeALL internals such as the Lyee identification policy of program variables, the generated program structure and the Lyee program execution control mechanism. Experience with LyeeAll has shown the need to acquire software requirements from relatively high level user-centric requirements. The meta-model of the LSRM presented in the bottom part of Fig. 9 has been used as a baseline paradigm model for the more abstract LURM construction shown in the top part of Fig. 9.

The central concept in the LSRM is called a Word. A Word corresponds to a program variable: input words represent values captured from the external world whereas output words are produced by the system by applying specific formulae. The execution of formulae is controlled by the Process Route Diagram (PRD). A PRD is composed of Scenario Functions $(S F)$, composed of Pallets, which are made of Vectors. In order to carry out the generated program control the function generates its own Words, such as the Action words related to Vectors and Routing words to distribute the control over the various $S F s$ of a $P R D$.

In order to comply with the Lyee paradigm, the LURM should be centred on a notion, which abstracts from the concept of Word. Obviously Words required by the Lyee processing mechanism are not relevant at this level. On the contrary, the concern is only with Domain words. Besides, there is a need to provide the requirements holder with a means to grasp a 'set of words' conceptually associated with one another. The notion of 'System interaction' is proposed for that purpose. An interaction delineates a number of input and output data, logically assembled together. Each word of an interaction is defined as a model element called Item by applying the Abstraction strategy (Fig. 8). The concept of Defined is proposed as an aggregation of

1 Lyee, which stands for GovernmentaL Methodolog $\underline{Y}$ for SoftwarE ProvidencE, is a methodology for software development used for the implementation of business software applications. Lyee was invented by Fumio Negoro. 
logically related Items thanks to the Aggregation strategy. The Specialisation strategy is applied in order to specialise the Item into Output and Input. An Output is produced by the system whereas the Input is captured from the user. In the same manner, the Input is specialised into Active and Passive. The former triggers the system actions whereas the latter represents values captured from the user. Similarly, the concept of Precedence Succedence Graph (PSG) is obtained by abstraction of the PRD concept from the LSRM. It specifies the ordering conditions between Defineds as the PRD do it with Words.

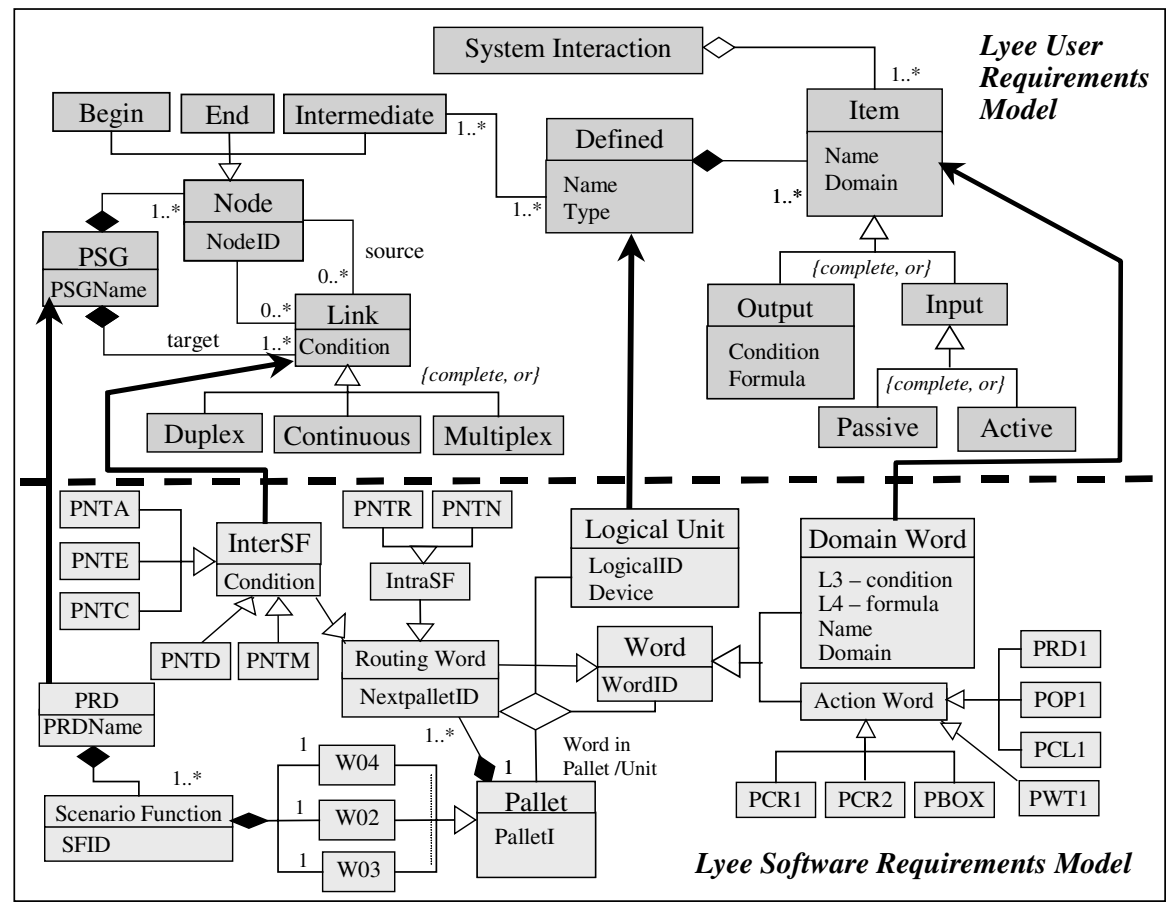

Fig. 9. Lyee Product Models for Software Requirements and for User Requirements

The process part of the LURM was defined by following the Pattern-based strategy (Fig. 7). A set of patterns has been defined to take into account different situations in the user requirements definition. Each pattern provides an advice to capture and formulate requirements. More about these patterns, their definition and application could be found in [24].

\section{Generic Features}

The paper demonstrates that meta-modelling remains the core technique in SME. All approaches presented above are based on meta-modelling. In the assembly-based SME approach every method chunk must be instance of a specific meta-model for 
modular methods [20]. The extension-based approach depends on the model of the method to extend which is itself instance of a specific meta-model, and proposes patterns to extend this model. The patterns are generated from the meta-patterns that are also defined at the meta level. The paradigm-based approach is generally based on meta-modelling.

All these approaches deal with the definition, instantiation, transformation or assembly of method models and meta-models. The corresponding method construction activities can be generalised by the means of a set of generic operators. As all these approaches explicitly separate the notions of product model and process model, we classify these operators in operators for process model construction and operators for product model construction. The former generalise the actions to be performed on the product models and deal with elements such as concepts, links and properties. The later generalise the actions to be performed on the process models and deal with elements such as intentions and strategies.

An other classification of these operators relates to the type of action they perform. Such a classification is as follows:

- Unification operators are used in order to unify the terminology of the models before their integration, extension or adaptation. They generally allow to rename different elements in the process and product models. Some examples of such operators are RENAME_CONCEPT, RENAME_INTENTION, etc.

- Transformation operators deal with the conversion of one type of product model element into another type. For example, the OBJECTIFY_LINK operator permits to transform a link between two classes of objects into a new class.

- Abstraction/instantiation operators deal with the different abstraction levels of the models. They can be used for the product model instantiation from a meta-model or its abstraction from another one.

- Specialisation/generalisation operators can be used for a connection of two product models having some concepts with similar semantics but different structures. A new concept can be generalised in order to preserve the two initial concepts in the integrated model.

- Aggregation/decomposition operators operate with different granularity levels and allow to combine or to split different product and process model elements as for example AGGREGATE_CONCEPTS or DECOMPOSE_SECTION.

- Addition operators allow to add supplementary elements (concepts, links, sections) in the product and process models in order to connect or to enhance them.

- Cancellation operators such as REMOVE_LINK or REMOVE_STRATEGY eliminate the inadequate elements from the product or process models.

We are aware of the fact that this list of operators is not an exhaustive one and we are currently working on it.

The last point that we propose to raise in this section concerns the requirements matching problem. The specificity of SME approaches is that they are requirementsdriven. Any method construction technique proposed by such an approach must take into account the definition of the method requirements and the selection of the solutions that satisfy them. As a consequence, the matching mechanism between the requirements model and the solution model is paramount. Our belief is that such a mechanism must include similarity measures. The method engineer needs to be able to measure the similarity of different elements from the process models like intentions, sections or entire maps as well as the similarity of different product models 
elements like concepts or links. Currently every approach integrated in our generic process model proposes its own manner to resolve the requirements matching problem. Our objective is to propose some generic process and product similarity measures that could be adapted or instantiated in different SME approaches.

\section{Conclusion}

In this paper we proposed a generic process model for SME. This process model allows us to capture different approaches for project specific method construction and to provide guidelines in order to assist the method engineer in the selection of the approach best fitting the project situation.

Our generic process model already contains three SME approaches that can be applied separately or combined in order to construct a new method or to adapt an existing one. As this model is defined as a map with associated guidelines it is possible to include other SME approaches in a rather simple manner. They can be integrated as different strategies to satisfy the intention Construct a Method (Fig. 1).

In order to provide a strong methodological support with our generic SME process model we propose a set of generic method construction operators. We are also working on different similarity measures which are necessary to evaluate the similarity between different method elements as well as for evaluating the matching conditions of a given method chunk with the method requirements.

Our future preoccupation is to complete this generic SME process model by integrating other approaches and to validate it through real projects. We will also continue refining the definition of the generic method construction operators and the metrics for process and product models similarity measurement. We will also consider the exploit of the distance measures supposing that in some cases they might be more suitable than those of similarity.

\section{References}

1. Benjamen A., Une Approche Multi-démarches pour la modélisation des démarches méthodologiques. PhD dissertation. University of Paris 1 - Sorbonne, 1999.

2. Böhlen M.H. Valid time integrity constraint. Report TR 94-30. 1994.

3. Brinkkemper S., M. Saeki, F. Harmsen, Assembly Techniques for Method Engineering. Proc. of CAiSE'98. Pisa Italy, 1998.

4. Cockburn A., Writing Effective Use Cases. Addison-Wesley, 2001.

5. Deneckere, R., Approche d'extension de méthodes fondée sur l'utilisation de composants génériques, $\mathrm{PhD}$ thesis, University of Paris 1-Sorbonne, 2001.

6. Deneckere, R., Souveyet, C., Patterns for extending an OO model with temporal features. Proceedings of OOIS'98 conference. Springer-Verlag, Paris (France), 1998.

7. Gehani N., Jagadish H.V. Ode as an active database: constraints and triggers. Proceedings of the $17^{\text {th }}$ VLDB, Barcelona, Spain, pp. 327-336. 1991.

8. Grundy, J.C., J.R. Venable, Towards an integrated environment for method engineering, Proc. IFIP WG 8.1 Conf. on 'Method Engineering', Chapman and Hall, pp 45-62, 1996.

9. Harmsen A.F., Situational Method Engineering. Moret Ernst \& Young, 1997.

10. Hofstede, A.H.M. Ter., Information modelling in data intensive domains, Dissertation, University of Nijimegen, The Netherlands 1993. 
11. Jacobson I., M. Christenson, P. Jonsson, G. Oevergaard, Object Oriented Software Engineering: a Use Case Driven Approach. Addison-Wesley, 1992.

12. Jarke M., C. Rolland, A. Sutcliffe, R. Domges, The NATURE requirements Engineering. Shaker Verlag, Aachen 1999.

13. Negoro, F. Methodology to Determine Software in a Deterministic Manner. Proceedings of ICH, Beijing, China, 2001.

14. Negoro, F. A proposal for Requirement Engineering, Proceedings of ADBIS, Vilnius, Lithuania, 2001.

15. Plihon V., J. Ralyté, A. Benjamen, et al. A Reuse-Oriented Approach for the Construction of Scenario Based Methods. Proc. of the ICSP'98, Chicago, US, 1998.

16. Prakash, N., On Method Statics and Dynamics. Information Systems. Vol.34, No.8, 1999.

17. Prakash, N., M.P.S. Bhatia. Generic Models for Engineering Methods of Diverse Domains. Proc. of CAISE'02, Toronto, Canada, LNCS Volume 2348, pp. 612., 2002.

18. Punter H.T., K. Lemmen, The MEMA model: Towards a new approach for Method Engineering. Information and Software Technology, 38(4), pp.295-305, 1996.

19. Ralyté J., C. Rolland, An Assembly Process Model for Method Engineering. Proceedings of the $13^{\text {th }}$ CAISE'01, Interlaken, Switzerland, 2001.

20. Ralyté J., C. Rolland, An approach for method reengineering. Proceedings of the $20^{\text {th }}$ International Conference on Conceptual Modelling, ER2001, Yokohama, Japan, 2001.

21. Ralyté J., Requirements Definition for the Situational Method Engineering, IFIP TC8/WG8.1 Working Conference on Engineering Information Systems in the Internet Context, Kanazawa, Japan, 2002.

22. Ralyté J., C. Rolland, V. Plihon, Method Enhancement by Scenario Based Techniques. Proceedings of the $11^{\text {th }}$ CAiSE'99, Germany, 1999.

23. Ralyté J., Reusing Scenario Based Approaches in Requirement Engineering Methods: CREWS Method Base. Proc. of the First Int. Workshop REP'99 (DEXA'99), Florence, Italy, September 1999.

24. Rolland, C. A User Centric View of Lyee Requirements. In New Trends in Software Methodologies, Tools and Techniques, H. Fujita, P. Johannesson (Eds.). IOS Press, Ohmsha, 2002.

25. Rolland, C., C. Souveyet, M. Moreno, An Approach for Defining Ways-of-Working, Information Systems Journal, 1995.

26. Rolland C., V. Plihon, J. Ralyté, Specifying the reuse context of scenario method chunks. Proc. of the $10^{\text {th }}$ CAISE'98, Pisa Italy, 1998.

27. Rolland, C., N. Prakash, A. Benjamen: A Multi-Model Vew of Process Modelling, Requirements Engineering Journal (4)(4), pp169-187, 1999.

28. Saeki, M., K. Wen-yin, Specifying Software Specification and Design Methods, Proc. CAISE'94, LNCS 811, Springer Verlag, pp 353-366, Berlin, 1994.

29. Saeki M., K. Iguchi, K Wen-yin, M Shinohara, A meta-model for representing software specification \& design methods. Proc. of the IFIP"WG8.1 Conference on Information Systems Development Process, Come, pp 149-166, 1993.

30. Snodgrass, I. Ahn. A ytaxonomy of time in databases. Proceedings of ACM SIGMOD conference. 1985. 